\title{
Gastric Ulcer, CTCAE
}

National Cancer Institute

\section{Source}

National Cancer Institute. Gastric Ulcer, CT CAE. NCI Thesaurus. Code C143504.

A disorder characterized by a circumscribed, erosive lesion on the mucosal surface of the stomach. 